Article

\title{
Personalized Assessment of Normal Tissue Radiosensitivity via Transcriptome Response to Photon, Proton and Carbon Irradiation in Patient-Derived Human Intestinal Organoids
}

\author{
Ali Nowrouzi ${ }^{1,2,3,4, *}$, Mathieu G. Sertorio ${ }^{5}$, Mahdi Akbarpour ${ }^{1,2,3,4}$, Maximillian Knoll 1,2,3,4, \\ Damir Krunic ${ }^{6}$, Matthew Kuhar ${ }^{7}$, Christian Schwager $1,2,3,4$, Stephan Brons ${ }^{3}$, \\ Jürgen Debus ${ }^{1,2,3,4}$, Susanne I. Wells ${ }^{5}$, James M. Wells ${ }^{7,8,9}$ and Amir Abdollahi ${ }^{1,2,3,4}$ (D) \\ 1 Heidelberg Ion-Beam Therapy Center (HIT), Department of Radiation Oncology, Heidelberg University \\ Hospital (UKHD), 69120 Heidelberg, Germany; mahdi.akbarpour@dkfz-heidelberg.de (M.A.); \\ m.knoll@dkfz-heidelberg.de (M.K.); C.Schwager@dkfz-heidelberg.de (C.S.); \\ juergen.debus@med.uni-heidelberg.de (J.D.); a.amir@dkfz-heidelberg.de (A.A.) \\ 2 German Cancer Consortium (DKTK) Core Center, Clinical Cooperation Units (CCU) Translational Radiation \\ Oncology and Radiation Oncology, National Center for Tumor Diseases (NCT), German Cancer Research \\ Center (DKFZ), 69120 Heidelberg, Germany \\ 3 Heidelberg Institute of Radiation Oncology (HIRO), National Center for Radiation Research in \\ Oncology (NCRO), German Cancer Research Center (DKFZ) and Heidelberg University Hospital (UKHD), \\ 69120 Heidelberg, Germany; Stephan.Brons@med.uni-heidelberg.de \\ 4 Division of Molecular and Translational Radiation Oncology, Heidelberg Medical Faculty (HDMF), \\ Heidelberg University, 69120 Heidelberg, Germany \\ 5 Cancer and Blood Diseases Institute, Cincinnati Children's Hospital Medical Center, Cincinnati, OH 45229, \\ USA; Mathieu.Sertorio@cchmc.org (M.G.S.); susanne.wells@cchmc.org (S.I.W.) \\ 6 Light Microscopy Facility, German Cancer Research Center, 69120 Heidelberg, Germany; \\ D.Krunic@dkfz-heidelberg.de \\ 7 Division of Developmental Biology, Cincinnati Children's Hospital Medical Center, Cincinnati, $\mathrm{OH} 45229$, \\ USA; Kuhv2r@gmail.com (M.K.); james.wells@cchmc.org (J.M.W.) \\ 8 Division of Endocrinology, Cincinnati Children's Hospital Medical Center, Cincinnati, OH 45229, USA \\ 9 Center for Stem Cell and Organoid Medicine, Cincinnati Children's Hospital Medical Center, Cincinnati, \\ $\mathrm{OH} 45229$, USA \\ * Correspondence: a.nowrouzi@dkfz-heidelberg.de; Tel.: +49-6221-56-34638
}

Received: 13 January 2020; Accepted: 14 February 2020; Published: 18 February 2020

\begin{abstract}
Radiation-induced normal tissue toxicity often limits the curative treatment of cancer. Moreover, normal tissue relative biological effectiveness data for high-linear energy transfer particles are urgently needed. We propose a strategy based on transcriptome analysis of patient-derived human intestinal organoids (HIO) to determine molecular surrogates for radioresponse of gastrointestinal (GI) organs at risk in a personalized manner. HIO were generated from induced pluripotent stem cells (iPSC), which were derived from skin biopsies of three patients, including two patients with FANCA deficiency as a paradigm for enhanced radiosensitivity. For the two Fanconi anemia (FA) patients (HIO-104 and 106, previously published as FA-A\#1 IND-iPS1 and FA-A\#2 IND-iPS3), FANCA expression was reconstituted as a prerequisite for generation of $\mathrm{HIO}$ via lentiviral expression of a doxycycline inducible construct. For radiosensitivity analysis, FANCA deficient and FANCA rescued as well as wtHIO were sham treated or irradiated with 4Gy photon, proton or carbon ions at HIT, respectively. Immunofluorescence staining of HIO for 53BP1-foci was performed $1 \mathrm{~h}$ post IR and gene expression analyses was performed 12 and $48 \mathrm{~h}$ post IR. 53BP1-foci numbers and size correlated with the higher RBE of carbon ions. A FANCA dependent differential gene expression in response to radiation was found ( $p<0.01$, ANOVA; $\mathrm{n}=107112 \mathrm{~h} ; \mathrm{n}=110048 \mathrm{~h}$ ). Pathways associated with FA and DNA-damage repair i.e., transcriptional coupled nucleotide excision repair, homology-directed
\end{abstract}


repair and translational synthesis were found to be differentially regulated in FANCA deficient HIO. Next, differential regulated genes were investigated as a function of radiation quality ( $R Q, p<0.05$, ANOVA; $\mathrm{n}=74212 \mathrm{~h} ; \mathrm{n}=55348 \mathrm{~h}$ ). Interestingly, a gradual increase or decrease of gene expression was found to correlate with the three main qualities, from photon to proton and carbon irradiation. Clustering separated high-linear energy transfer irradiation with carbons from proton and photon irradiation. Genes associated with dual incision steps of TC-NER were differentially regulated in photon vs. proton and carbon irradiation. Consequently, SUMO3, ALC1, POLE4, PCBP4, MUTYH expression correlated with the higher RBE of carbon ions. An interaction between the two studied parameters FA and RQ was identified $(p<0.01$, 2-way ANOVA $n=476)$. A comparison of genes regulated as a function of FA, RQ and RBE suggest a role for p53 interacting genes BRD7, EWSR1, FBXO11, FBXW8, HMGB1, MAGED2, PCBP4, and RPS27 as modulators of FA in response to radiation. This proof of concept study demonstrates that patient tailored evaluation of GI response to radiation is feasible via generation of $\mathrm{HIO}$ and comparative transcriptome profiling. This methodology can now be further explored for a personalized assessment of GI radiosensitivity and RBE estimation.

Keywords: human intestinal organoids (HIO); radiotherapy; gastrointestinal (GI) toxicity; particle therapy; carbon ions; personalized medicine

\section{Introduction}

Radiosensitivity of gastrointestinal (GI) organs such as the esophagus, duodenum, and rectum, are critical, e.g., for the curative treatment of thoracic tumors, and pancreatic or prostate cancer. Moreover, stomach and small bowel toxicity is a major limitation for high dose palliative radiotherapy on lumbar bone metastasis or retroperitoneal lymph nodes. Empirical data on normal tissue complication probability (NTCP) considering radiation dose and volume such as QUANTEC (quantitative analysis of normal tissue effects in the clinic) has substantially improved radiotherapy [1]. However, these approaches do not consider the broad spectrum of inter-individual heterogeneity in GI response to radiation that may result from genetic predisposition of the patient. Ionizing radiation (IR)-induced gastrointestinal (GI) toxicity imposes a challenge for the radiotherapy of cancer patients [2-4]. These toxic effects are classified as acute, developing within days or weeks of radiation exposure, or as chronic, developing months and years after treatment [5]. Acute effects related to direct DNA damage, p53 activation, cell cycle arrest, senescence and apoptosis most likely also lead to the development of chronic effects such as the loss of the epithelial integrity, activation of inflammatory processes which may further promote damage to the vascular and induced connective tissue fibrosis, atrophy and chronic tissue dysfunction. This underlines the importance of investigating early and late effects of radiation toxicity coordinately [5].

For understanding the causal connection of acute and late effects of IR-induced GI toxicity most ideally, experiments have to be performed in a tissue specific context which makes animal experiments indispensable at present. Such animal experiments are difficult to design and perform since long observation times and large animal numbers are required. With the engineering of human pluripotent-stem-cell-derived gastrointestinal organoids [6-8] a highly promising technology is available which can enable the rapid monitoring of personalized acute and chronic effects of radiation on the GI tract in vitro. Protocols for establishing organoids from pluripotent cells have been reported for many organs (intestine, stomach, pancreas, brain, liver, eyes) [9,10]. Organoids consist of many types of cells which differentiate from progenitor stages during organoid development and also have shown to have the ability to recapitulate normal tissue functions [10-13]. Thus, organoids resemble human organs and, therefore, constitute an attractive technology to complement in vivo animal studies for understanding mechanism involved in human tissue repair, regeneration and therapy response/toxicity in an authentic context $[9,10]$. 
Normal tissue is aimed to be spared by high-precision irradiation with particles. However, compared to conventional photon irradiation, uncertainties of relative biological effectiveness (RBE) of protons, and even more so for heavier ions with higher linear energy transfer (LET) such as carbon radiotherapy exist $[14,15]$. We here have evaluated the feasibility of human intestinal organoids (HIO) to recapitulate patient radio-sensitivity. Moreover, with the advent of high-precision irradiation using protons and heavier ions such as carbon ions at HIT [14,16], we sought to evaluate the feasibility of this approach to decipher the transcriptome response as a surrogate for RBE of different radiation qualities (RQ). To evaluate whether potential differences in the transcriptional response to radiation is influenced by the genetic predisposition of the patients we have compared radiation-associated gene expression patterns in normal to FANCA deficient HIO which were derived from iPSC of individuals with FA [17]. The sensitivity of FA patients to chemotherapy agents involved in DNA damaging processes such as cisplatin and mitomycin $C$ is well known. However, although adjuvant radiation therapy is recommended for high stage tumors, systematic identification of safe radiation therapy (RT) dose levels for FA cancer patients who receive RT have not been reported [18]. As a proof of concept study, this work demonstrates the feasibility of individual assessment of radio-sensitivity by ex-vivo irradiation of $\mathrm{HIO}$ combined with a series of surrogates of radiation response including transcriptome and radiation-induced nuclear foci (RIF) formation.

\section{Results}

\subsection{Detection and Quantification of Radiation-Induced Foci (RIF) in Pluripotent Derived Human Intestinal Organoids}

Human intestinal organoids ( $\mathrm{HIO}$ ) were generated from the $\mathrm{H} 1$ embryonic stem cell line (H1-HIO) and two independent (104-HIO and 106-HIO) previously described induced pluripotent stem cell (iPSC) lines [17]. The 104- and 106-HIO were generated from patient specific iPSC which were derived from keratinocytes (104-HIO) or fibroblasts (106-HIO) biopsies of Fanconi anemia patients carrying a mutation in the FANCA gene. The H1-HIO was derived from the H1 embryonic stem cell line cells and is considered as the normal healthy phenotype (Figure 1a). FANCA expression is required for the efficient generation of iPSC [19,20] and HIO [17]. Therefore, prior to reprogramming, iPSC patient biopsies had been transduced with lentivirus expressing the wild type FANCA gene under doxycycline (DOX)-inducible control to rescue the FANCA deficiency. The established 104- and 106-HIO maintain characteristics of FA deficient cells in response to cytotoxic agents [17] in the absence of DOX-induced complementation with FANCA. In the following we will refer to HIO in which (DOX)-induced expression of FANCA expression was induced by doxycylin (DOX) as FANCA rescued and in the absence of DOX as FANCA-deficient HIO.

From each line single organoids were embedded in Matrigel in 96-wells and subjected to three different radiation qualities (Figure 1b). HIOs derived from all three pluripotent lines were irradiated with 4Gy physical doses of photon as well as clinical mixed beam field (SOBP) irradiation with proton or carbon ions (Figure 1c). A calculation of the local effect model (LEM) was performed predicting RBE doses of proton and carbon compared to photon, respectively (Supplementary Figure S1). One hour after radiation exposure, immunostaining was performed on HIO cryosections using an antibody against 53BP1 which accumulates at radiation-induced DNA-double-strand breaks (DSB) forming radiation-induced foci (RIF) (Figure 1d). Foci numbers and size were imaged by fluorescence microscopy and quantified with in house developed ImageJ macros (see methods). Compared to the non-irradiated control we detected a higher 53BP1-foci number per cell in carbon compared to proton irradiated HIO from both the FANCA proficient and deficient background (Figure 1d). Moreover, we determined the average size of 53BP1-foci in wt and FA-deficient HIO which were irradiated with proton and carbon beams respectively. This analysis shows that as previously described irradiation with carbon ions results in larger 53BP1-foci as compared to photon and proton beams which is considered an indicative for more complex DNA damage patterns induced by high-LET irradiation (Figure 1d). 
a.

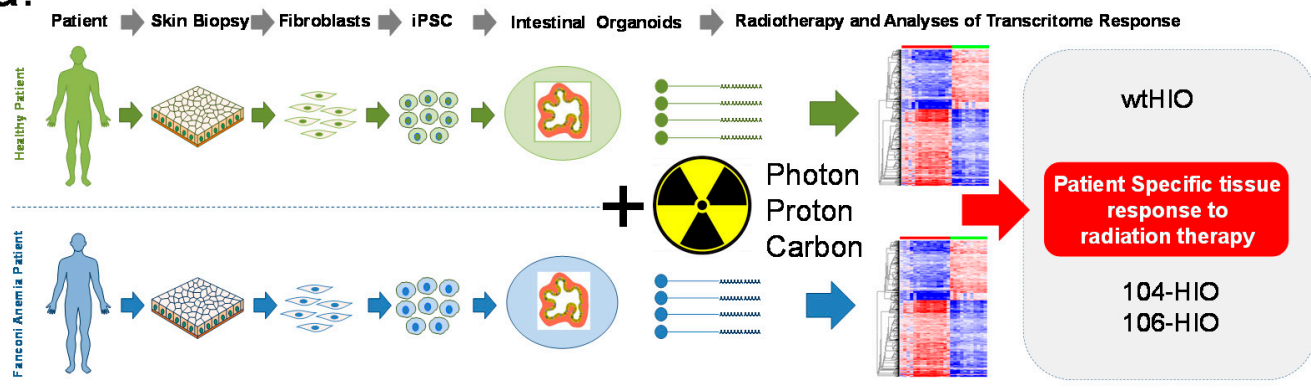

b.

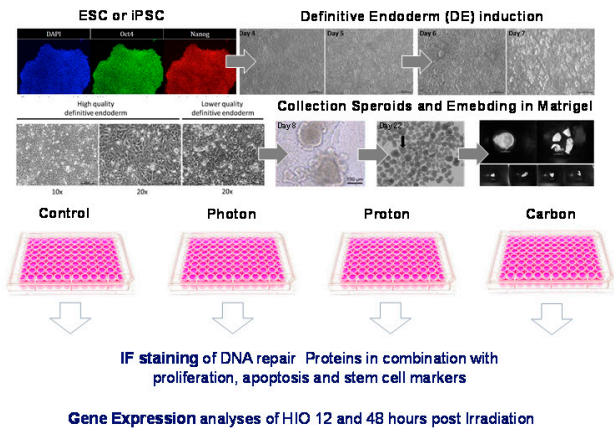

C.

d.
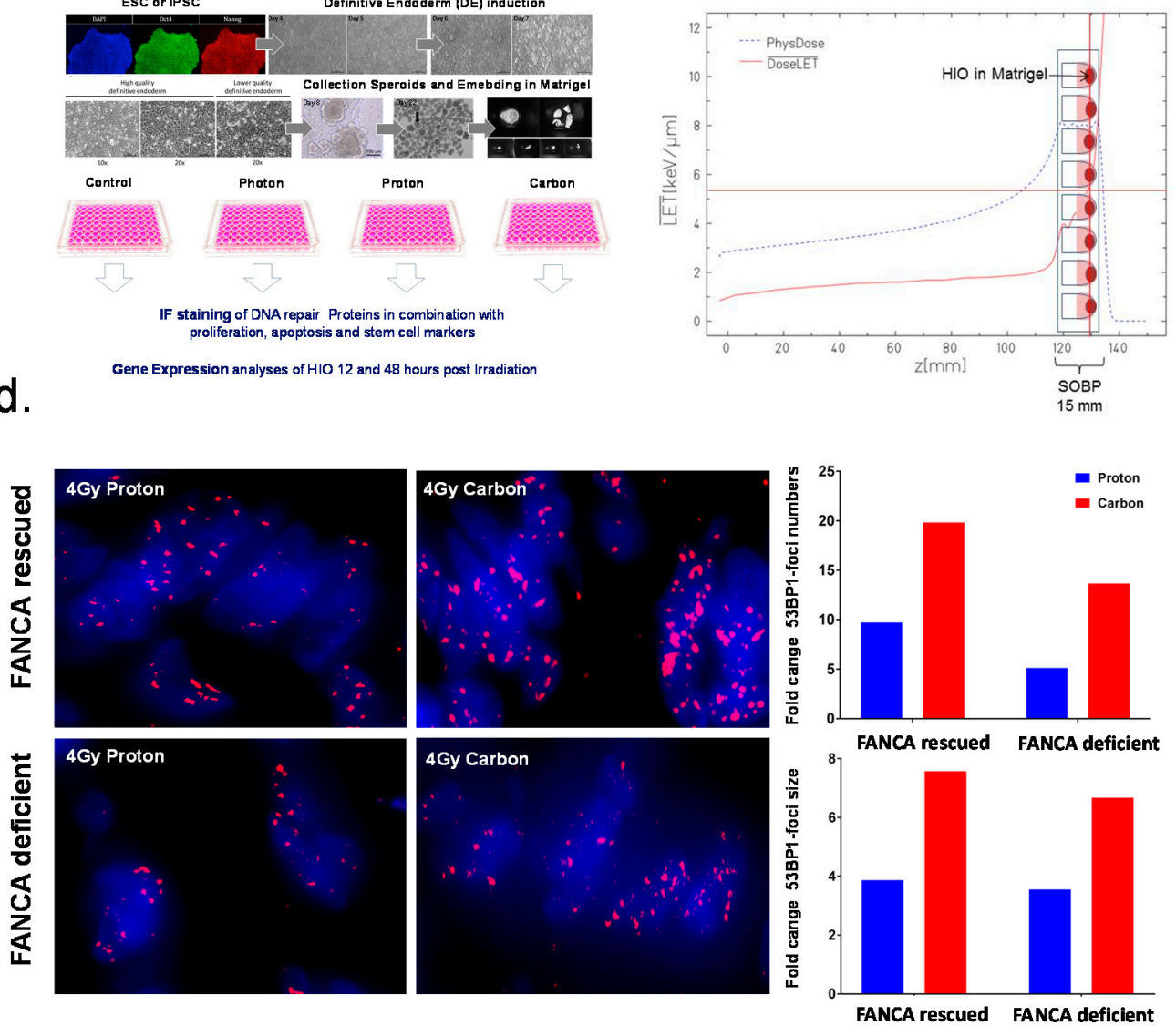

Figure 1. Irradiation of pluripotent derived human intestinal organoids with photon, proton and carbon irradiation. (a) Schematic outline on how organoids can be used to identify molecular surrogates of normal tissue radiation sensitivity and toxicity (b) Experimental outline of the study. Images of different stages of organoid development were kindly provided by the Center for Stem Cell and Organoid Medicine, Cincinnati. Human intestinal organoids (HIO) were generated as described previously and then embedded singularly in Matrigel in 96-plates. Following exposure to $4 \mathrm{~Gy}$ photon, proton and carbon irradiation HIO were cryo frozen for IF staining and stored for subsequent RNA isolation. (c) LEM prediction of carbon ions and positioning of organoids for irradiation. (d) Radiation-induced repair foci were visualized in cryo sections of irradiated HIO with IF stainings of 53BP1. The intensity of radiation-induced damage in $\mathrm{HIO}$ was quantified by measuring the 53BP1 numbers and size per cell. Represented values are normalized to background levels of 53BP1-foci. 
2.2. Comparative Gene Expression Analyses between HIO Derived from Healthy Individuals with Fanconi Anemia in Response to Radiation

For analyzing the transcriptional response of $\mathrm{HIO}$ to ionizing radiation, 3 organoids on average were embedded in Matrigel in 96-wells. One 96-well plate served as the non-irradiated control and three 96-well plates were irradiated with 4Gy photon, proton and carbon irradiation separately (Supplementary Table S15). Gene expression analyses were performed 12 and $48 \mathrm{~h}$ after irradiation. First, we confirmed that the 104-HIO and 106-HIO expressed higher levels of FANCA in the presence of doxycycline (Supplementary Figure S2a,b). Next we determined whether baseline gene expression differences can be observed in HIOs with a functional and non-functional Fanconi anemia pathway. Therefore, the expression level of genes in 104-HIO FANCA rescued vs. FANCA-deficient and 106-HIO FANCA rescued vs. FANCA-deficient were compared (Supplementary Figure S2c). Both in the 104-HIO and 106-HIO only minor gene expression differences could be detected between the presence and absence of the FANCA gene which could not be linked to the Fanconi anemia pathway directly (Supplementary Figure S2d,e). In comparison to the H1-HIO, gene expression differences were predominately detectable between the 104-HIO obtained from iPSCs derived from keratinocyte whereas the gene expression pattern of the 106-HIO obtained from iPSCs derived from fibroblast was more similar to the H1-HIO (Supplementary Figure S3a). For the comparison of gene expression differences between FA proficient and deficient $\mathrm{HIO}$ only the 106-HIO was used. Interestingly in response to irradiation which acts as cytotoxic agent inducing DNA double strand breaks (DSB), a comparison between the $\mathrm{H} 1-\mathrm{HIO}$ and the 106-HIO revealed that in the presence of a functionally active FANCA gene (106-HIO FANCA rescued) the global DNA repair and metabolism-associated transcriptional response programs are more similar to the H1-HIO. In contrast, the FANCA-deficient 106-HIO (106-HIO FANCA-deficient) showed a differential transcriptional response (Figure 2a,b, Supplementary Figure S3b,c). A comparison of gene expression values between healthy HIO and FA-derived 106-HIO (106-HIO FANCA rescued and 106-HIO FANCA-deficient) identified genes ( $p$ $<0.01$, ANOVA; $\mathrm{n}=1071$ 12h; $\mathrm{n}=1100$ 48h) which showed a differential regulation in response to irradiation (Supplementary Table S1). The 106-HIO FANCA rescued gene expression signature was more similar to H1-HIO were as in 106-HIO FANCA-deficient harbored greater differences as shown by hierarchical cluster (Manhattan distance) analysis (Figure 2a,b, Supplementary Figure S3c). From these genes 660 genes showed a significant $(p<0.01, T$-test) gene expression difference after $12 \mathrm{~h}$ and 789 genes after $48 \mathrm{~h}$ in 106-HIO FANCA-deficient compared to the H1-HIO (Figure 2a,b; Supplementary Table S2). The top functional categories which were significantly affected by differential gene regulation in irradiated FANCA-deficient 106-HIO FANCA-deficient after $12 \mathrm{~h}$ were processing of pre-mRNA and rRNA ( $p<0.006$; CSNK1E, DDX49, EXOSC7, NOC4L, NOP14, PWP2, RPL27A, RPL9, RPS14, RPS15A, RPS25, RPS4X, RPS9, SIK1, WDR18, WDR46) and dual incision in transcription coupled nucleotide excision repair ( $p<0.02$; ERCC1, ERCC2, GTF2H4, POLD2, POLE2, POLR2J, POLR2K, PRPF19). After $48 \mathrm{~h}$ genes regulating FOXO-mediated transcription $(p<0.001$; BCL6, CCNG2, CDKN1A, CREBBP, NPY, RBL2, YWHAB) and targets of SUMO E3 ligases ( $p<0.02$; AAAS, CREBBP, DNMT3B, NCOR2, NR1I2, NR2C1, NSMCE1, NUP153, NUP160, NUP37, NUP54, PIAS2, SMC3, WRN, ZBED1) were specifically differentially regulated in FA-deficient HIO. 


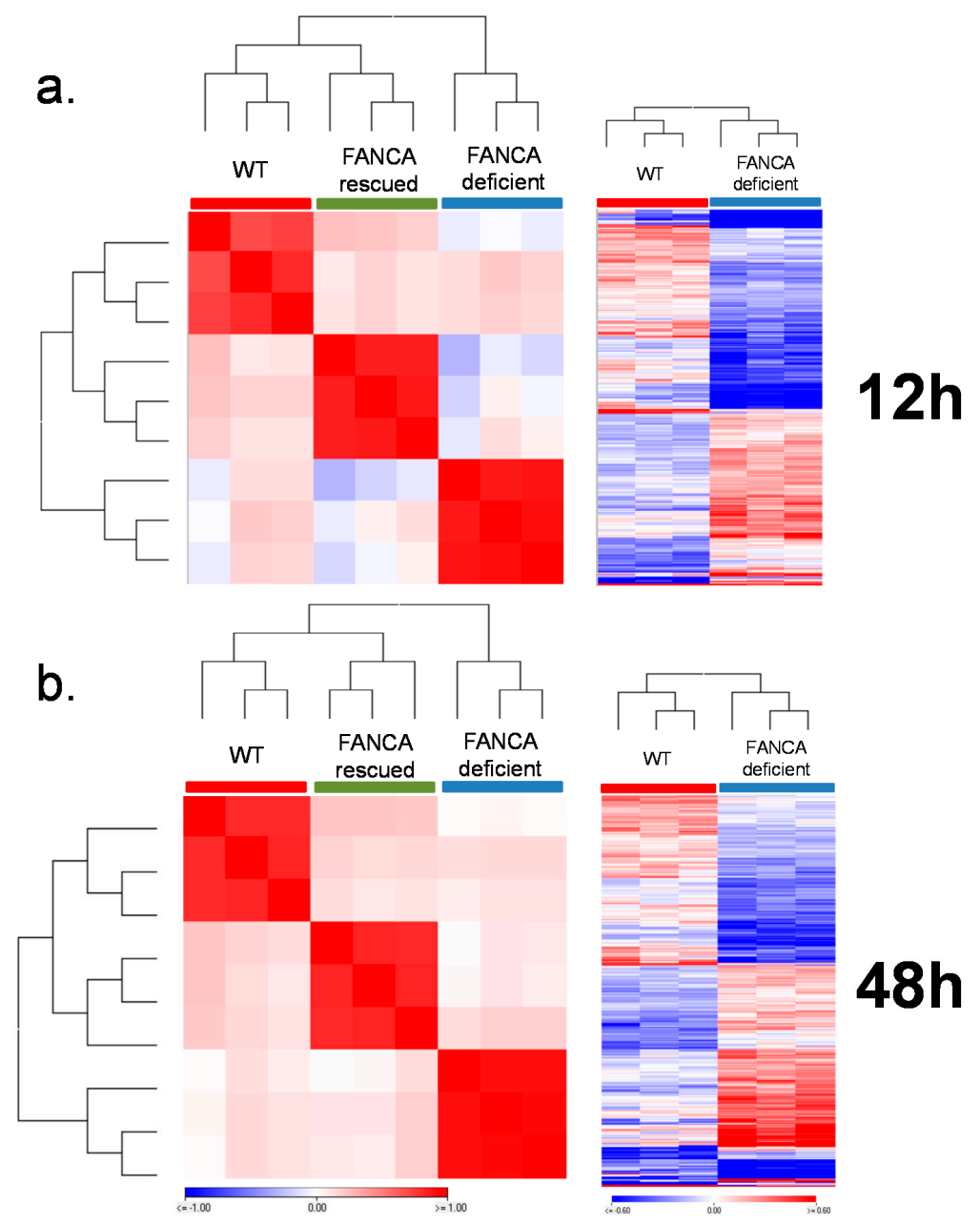

Figure 2. Transcriptional patterns in response to photon, proton and carbon irradiation in wild type and FANCA-deficient HIO derived from healthy and Fanconi anemia individuals. The transcriptional status of genes related to the Fanconi anemia pathway was analyzed in the HIO-106 line 12 (a) and $48 \mathrm{~h}$ (b) post exposure to photon, proton and carbon ions. The Pearson correlation between wt and FANCA rescued and FANCA-deficient cells derived from FA patients clearly distinguishes FANCA-deficient $\mathrm{HIO}$ from HIO which express intact FANCA. For each time point gene expression values between healthy HIO and FA-derived 106-HIO (FANCA-deficient and FANCA rescued) were identified $(p<$ 0.01 , ANOVA; $\mathrm{n}=107112 \mathrm{~h} ; \mathrm{n}=110048 \mathrm{~h}$ ) which are differentially regulated. All radiation qualities were combined for this analysis.

FANCA-Deficient HIO Show a Differential Gene Expression Signature of DNA Damage Response Relevant Genes Compared to FANCA Proficient HIO

A comparison of 106-HIO FANCA rescued and 106-HIO FANCA-deficient identified 224 DDR-associated genes which were differentially $(p<0.05, T$-test) regulated in the 106-FANCA-deficient HIO (Supplementary Table S3). From these genes 50\% were also identified to distinguish the H1-HIO from the 106-HIO FANCA-deficient ( $p<0.05$, ANOVA). Generally, both after 12 and $48 \mathrm{~h}$ post exposure to IR core components of the transcription coupled nucleotide excision repair machinery (TC-NER) as well as genes involved in the regulation of translational synthesis (TLS) and homology-directed repair showed differential regulation specific for 106-HIO FANCA-deficient (Figure 3a; Supplementary Table S4). After $12 \mathrm{~h}$ the strongest genes which were specifically upregulated in 106-HIO FANCA-deficient were SFN, POLD4, CDKN1A, TRIM25, and HIST1H2BE (Figure 3b). The strongest downregulated genes were dominated by genes which specifically regulate the transition form G1-G1/S phase (CDC25B, CDK6, MCM2, CDK4, CDKN1B, MCM7) and separation of sister chromatids at the mitotic spindle 
checkpoint (PTTG1, CENPF, RCC2, AURKB, CKAP5) (Figure 3b). Further we detected a differential regulation of Fanconi anemia-related genes ( $p<10-13$; ATRIP, BRCA1 EME1, ERCC1, FANCB, FANCC, FANCD2, FANCE, FANCG, RPA1, RPS27A, UBA52, UBE2T) and translesion synthesis regulating genes ( $p<$ 10-16; CUL4A, MAD2L2, POLD1, POLD2, POLD3, POLD4, POLE2, RFC3, RFC5, RPA1, RPS27A, UBA52, UBA7) in 106-HIO FANCA-deficient (Supplementary Figure S4). Interestingly all except for EME1 and POLD4 were downregulated (Supplementary Table S3) in 106-HIO FANCA-deficient. After $48 \mathrm{~h}$ the most significant genes which were specifically upregulated in 106-HIO FANCA-deficient were CDKN2A, CDKN1A, YWHAE which are related to the development of cellular senescence. The top downregulated genes were HIST1H2BD, HIST1H4H, SUMO2, and RAD51AP1 involved in regulating homology-directed repair by homologous recombination or single strand annealing. Further FOS a component of the SMAD3/SMAD4/JUN/FOS complex and APEX1 a AP endonuclease class 1 enzyme involved in DNA base excision repair (BER) pathway of DNA lesions induced by oxidative and alkylating agents were specifically downregulated in 106-HIO FANCA-deficient. SKP2 and MCM2 both involved in the switching of replication origins to a post-replicative state were also specifically downregulated in 106-HIO FANCA-deficient (Figure 3b).

a.

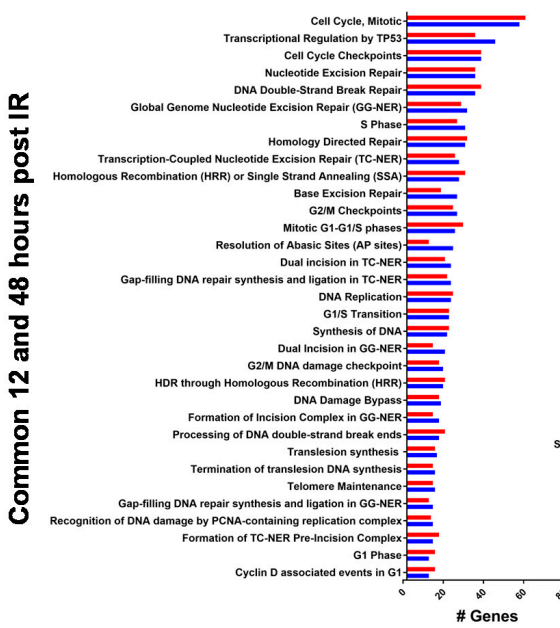

c.

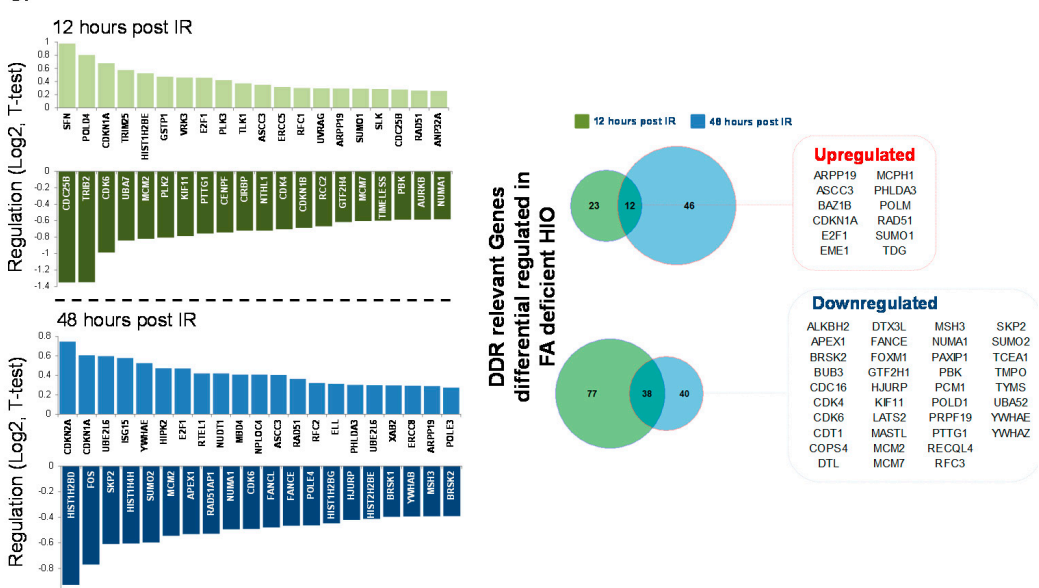

b.
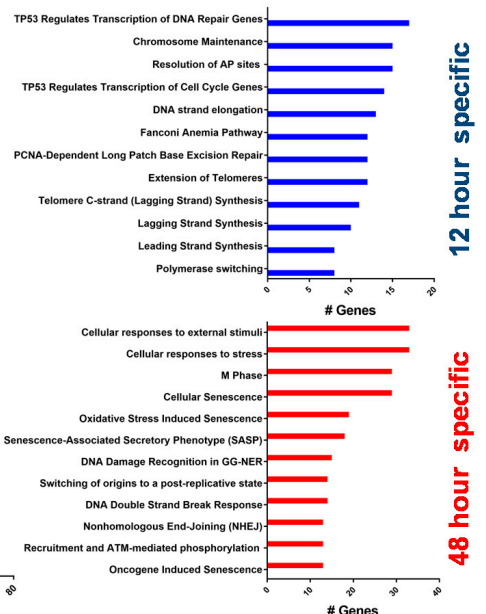

这$$
\text { . }
$$

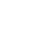




\subsection{Photon, Proton and Carbon Radiation Induce Common and Radiation Quality Specific Gene Expression} Signatures in Irradiated HIOs

A Pearson correlation on normalized gene expression data revealed that independent of the radiation quality used, the most significant gene expression differences were detectable between 12 and $48 \mathrm{~h}$ in all three HIO tested (Figure 3a,b and Appendices A and B). Hierarchical clustering resulted in two main cluster branches which were composed of distinct gene expression regulation patterns after 12 and 48 h post IR (Supplementary Figure S5a). A summary of common transcriptional signatures of $\mathrm{HIO}$ in response to photon, proton and carbon irradiation is presented in the Appendices A and B.

Interestingly following a Pearson correlation we observed transcriptional differences between photon and particle (proton and carbon) irradiated HIO indicating differences in acute and late response of HIO exposed to photon compared to proton and carbon beams (Supplementary Figure S5a). This sub-clustering was more pronounced $12 \mathrm{~h}$ compared to $48 \mathrm{~h}$ post irradiation were the transcriptional response of $\mathrm{HIO}$ to carbon beams showed a unique and separate pattern compared to photon and proton beams after 48 h (Supplementary Figure S5a). After 12 h 742 genes (Supplementary Table S5) were differentially regulated ( $p<0.05$, ANOVA) between photon compared to proton and carbon beams (Supplementary Figure S5a,b). Among the top functional categories affected by differentially regulated genes were genes involved in dual incision steps of transcription coupled nucleotide excision repair (TCEA1, ERCC5, POLR2B, ERCC3, POLR2D, UBC, MCRS1, XAB2, REM1, POLR2I, DDB1). After $48 \mathrm{~h}$ the transcriptional response of photon and proton beams was more similar. A total of 553 genes (Supplementary Table S5) could be identified which were differentially regulated $(p<0.05$, ANOVA) between photon and proton compared to carbon beams. Carbon ion beams have a higher LET and superior RBE in comparison to photon and proton ions were as the RBE of Proton is similar to Photon (Supplementary Figure S7a,b). Therefore, we investigated whether specific gene expression signatures correlate and are unique in response to carbon irradiation by performing pavlidis template matching (PTM) analyses on gene expression data of the 12 and $48 \mathrm{~h}$ time points. After $12 \mathrm{~h}$ we found 230 transcriptional signatures which were significantly $(p<0.01)$ correlated with the higher RBE of carbon ions and 225 which were anti-correlated (Figure 4a; Supplementary Table S6). Among the correlated genes cellular processes regulated by sterol regulatory element-binding transcription factor (LSS, FASN and FDPS) were overrepresented. Expression of SUMO3 a small ubiquitin-related modifier which accumulates at DSB and in interaction with the SUMO E3 ligases PIAS1 and PIAS4 regulates DNA repair of DSB [21] correlated with higher RBE of carbon ions. Further the DNA helicase CHD1L also known as ALC1 [22] and the DNA polymerase POLE4 [23] also correlated to higher RBE of Carbon ions. PCBP4, which is activated by $\mathrm{p} 53$, and which suppresses cell proliferation by inducing apoptosis and cell cycle arrest $[24,25]$ correlates with the higher RBE of Carbon ions as well as the protein translation regulating genes (RPL34, RPS15, RPS28, MRPS34, MTRF1L, MRRF, ERAL1, KARS, SARS2). Among the top functional categories which were anti-correlating with a higher RBE of carbon beams were P53 and FOXO regulated cell death genes (BCL6, CNOT8, EP300, FAS, FOXO3, GADD45A, JMY, JUN, L3MBTL, MAPKAPK5, PPP2R5C, PRKAG1, SGK, SGK1, TNFRSF10D, UBC). Genes regulating membrane trafficking USO1, PRKAG1, ARL1, COG6, FCHO2, COG1, SCARB2, SEC31A, VAMP4, VPS25, SEC22A, TRIP11, YIPF6, GCC2, AAK1 were also anti-correlated with the superior RBE of Carbon beams. After 48 h 301 transcriptional signatures were significantly $(p<0.01)$ correlated with a higher RBE of carbon beams and 343 genes were anti-correlated (Figure 4a; Supplementary Table S6). The most dominant correlated gene was the adenine DNA glycolase MUTYH which is involved oxidative DNA damage repair by signaling apoptosis after the induction of single strand breaks [26]. Other genes involved in base excision repair by translational synthesis (RFC2, RFC1, HIST1H2BE, HIST1H2BC) were also correlated with carbon beams. Anti-correlated genes were the ubiquitin protein ligase FBXW7 and other members of the E2 ubiquitin-conjugation enzyme family (UBE2E1, CTR9, HIP2, UBE2H, SELS). 
a.

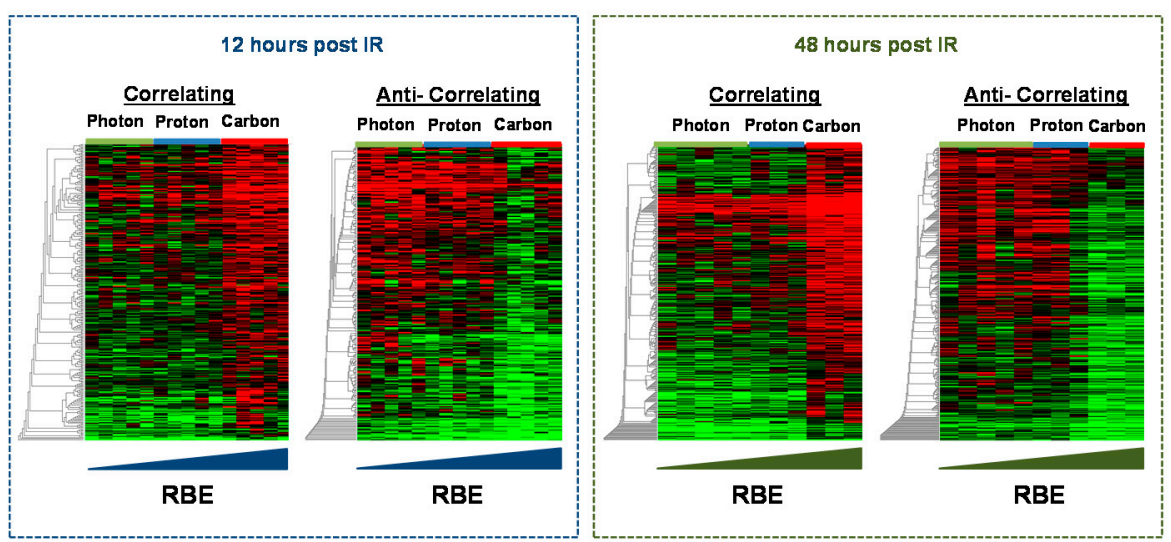

b. 12 hours post IR

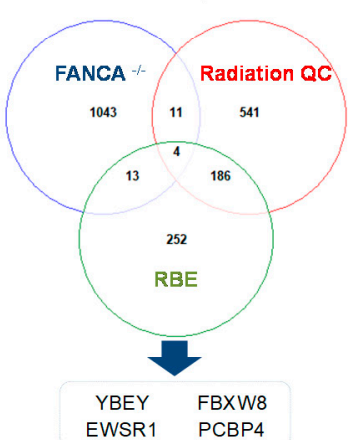

48 hours post IR

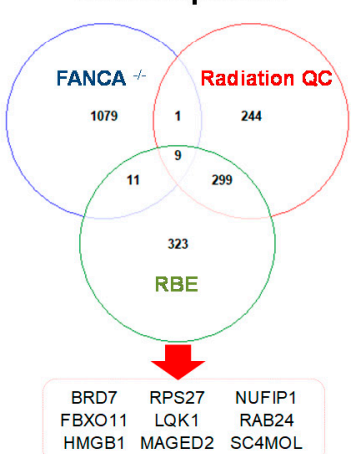

C.

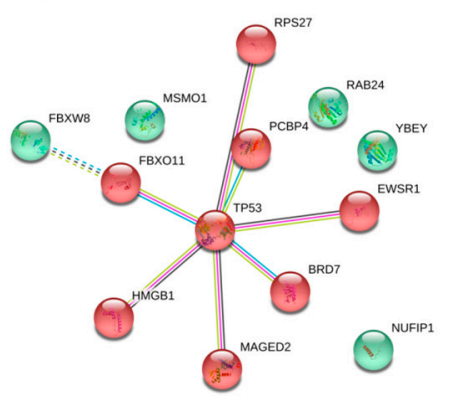

Figure 4. Gene expression changes as a function of Fanconi anemia, radiation quality and RBE. (a) gene expression heatmaps of genes which are correlated or anti-correlated with the higher RBE of carbon ions for the 12 and $48 \mathrm{~h}$ timepoint $(p<0.05, \mathrm{PTM})$. (b) The Venn diagram represent the overlap of genes modulated by FA, radiation qualities (RQ), and relative biological effectiveness (RBE) status for each timepoint resembling the core set of genes modulating FA response to radiation. (c) Genes which were modulated as a function of FA, RQ and RBE were analyzed with the STRING Search Tool for the Retrieval of Interacting Genes/Proteins. Represented are genes which interact with each other over the TP53 access.

\subsection{Identifying the Role of the FA Pathway in Response to Different Radiation Qualities and Determining Molecular Surrogates of Normal GI Tissue Response in FA Patients}

Since differential gene regulation patterns were identified in HIO based on the FANCA status and the applied radiation qualities we further analyzed whether the interaction of genetic predisposition, radiation quality and superior RBE of carbon beams results in distinct transcriptional signatures identifying genes which potentially could resemble surrogates for a personalized assessment of normal tissue radiosensitivity of FA patients. First a two-way-ANOVA was performed to specifically assess the interaction of the FA and radiation quality effect on irradiated HIO with no functionally active FA pathway. These analyses revealed that particular genes were differentially regulated in response to the different radiation qualities used and the FANCA expression status. A total of 476 genes (Supplementary Figure S8; Supplementary Table S7) were significantly $(p<0.01)$ correlated to FANCA status linking both the FA and radiation quality effect. The Fanconi anemia pathway genes FANCB, POLN, DCLRE1A, UBA52, and FANCF genes were significantly $(p<0.01, T$-test) downregulated after radiation only in FANCA-deficient HIO lines. Genes involved in the negative regulation of MET Proto-Oncogene Receptor Tyrosine Kinase signaling (LIG1, UBA52 and SH3GL2) were also specifically 
downregulated ( $p<0.01, T$-test) in response to the radiation qualities in FANCA-deficient HIO lines. In contrast NOTCH1 signaling genes (FBXW7, CUL1, UBA52, and HDAC7) were specifically upregulated $(p<0.01, T$-test $)$ in response to IR in FANCA-deficient HIO lines. Second we compared three sets of gene lists which were defined as (I) differentially regulated in 106-HIO FANCA-deficient cells compared to healthy cells, (II) differentially regulated in comparison of radiation qualities and (III) differentially regulated with higher RBE to define a core set of genes resembling potential surrogates of FA-associated normal tissue radio-sensitivity and/or -toxicity. Based on this analysis for each time point after radiation a core set of genes was defined which captures both the FA phenotype as well as the effect of different radiation qualities as well as superior RBE of carbon ions (Figure $4 \mathrm{~b}$ ). These core genes a predominantly involved in p53-regulated signaling processes (Figure 4c).

\section{Discussion}

The identification of biomarkers for accessing radiation-induced normal tissue toxicity has important implications for personalized radiation therapy. Especially in the context of pathogenic genetic predispositions of cancer patients, cell-based models are required which can potentially unmask molecular determinants of normal tissue toxicity of individual patients rapidly and efficiently. Organoids derived from tumor biopsies or from induced pluripotent stem cells comprise a promising technology to close this gap and to analyze personalized molecular determinants of radiation-induced tissue toxicity. Their multicellular organization and organ-like function provides unique advantages over 2D cell-based systems and potentially animal models.

We reasoned that analyzing gene expression signatures of organoids in response to radiation could define molecular surrogates of normal tissue toxicity. To evaluate our hypothesis human intestinal organoids were generated from iPSC and subjected to three different radiation qualities (photon, proton, carbon) at physical isodoses of $4 \mathrm{~Gy}$ to analyze their gene expression patterns post radiation. Therefore, two different time points after radiation were chosen (12 and $48 \mathrm{~h}$ ) to generate sequential gene expression signatures potentially resembling acute and late phases of radiation-induced toxicity. As a paradigm for the response of a highly sensitive tissue and for the testing of genetic predispositions in transcriptional radiation response we have compared the gene expression signature of HIO generated from iPSC of healthy individuals and Fanconi anemia patients carrying a mutation in the FANCA gene in response to radiation. Fanconi anemia is a rare genetic disease which is characterized by an impaired response to DNA damage and in particular a deficiency to repair DNA interstrand crosslinks (ICL). FA patients have a significantly increased risk of developing solid cancers, with a $28 \%$ cumulative incidence of solid cancers by the age of 40 . The high sensitivity to chemotherapy agents (particularly cisplatin and mitomycin C) is known and, therefore, often avoided in post-operative treatments. Adjuvant radiation therapy is recommended to treat patients with high stage tumors but sensitivity to radiation therapy is not well characterized on the molecular level in FA patients to the present, with FA patients showing different level of radio-sensitivity.

In comparison to non-irradiated controls independent of the genetic background or radiation quality, common gene expression signatures of irradiated $\mathrm{HIO}$ could be defined by an upregulation of p53-regulated processes and a downregulation of ATR-mediated stress response $12 \mathrm{~h}$ post IR. Gene expression patterns after $48 \mathrm{~h}$ affected significantly more functional categories compared to transcriptional alterations after $12 \mathrm{~h}$, showing that signaling cascades associated with the response of radiation sequentially increase in complexity after IR. The predominant molecular processes affected by gene expression changes after $48 \mathrm{~h}$ were associated with a downregulation of cell cycle checkpoint genes, regulation of the mitotic spindle checkpoint, separation of duplicated chromosomes such as components of the anaphase promoting complex/cyclosome (APC/C). This may indicate that mitotic cell division is delayed at the level of spindle checkpoint and kinetochore attachment [27] which is required for the separation of sister chromatids during DNA replication [28,29]. We cannot rule out that this common program is due to the exposure of the HIO to relatively high doses of $4 \mathrm{~Gy}$ which could resemble gene signatures correlated with toxic levels of radiation. Nevertheless, these 
distinct transcriptional changes in response to radiation manifest a multilevel interplay between DNA damage and pathways associated with protein turnover with the aim of restoring tissue hemostasis in response to radiation-induced cellular stress. Based on the gene expression analyses it is likely that these processes are regulated at the level of p53 response, protein translation, ubiquitination and SUMOylation to control mitotic cell division by common cellular stress response mechanisms.

The FA-derived HIO under normal non-irradiated conditions showed a transcriptional phenotype comparable to HIO derived from healthy patients. Similar to mouse models of FA [30] upon exposure to genotoxic agents such as radiation FA-deficient HIO showed a transcriptional pattern which is clearly distinguishable from genetically healthy conditions. FA-deficient HIO showed a differential regulation of genes involved in processing or pre-mRNA and rRNA, dual incision steps occurring during TC-NER, translational synthesis, homology-directed repair and FA pathway genes after 12 $\mathrm{h}$ sequentially switching to the differential regulation of FOXO-mediated transcription and targets of SUMO E3 ligases after $48 \mathrm{~h}$. Very recently the FA disease has been linked to defects in ribosomal biogenesis on the level of rRNA processing [31] which could explain why genes involved in such processes are differentially regulated in FA deficient $\mathrm{HIO}$ in response to radiation. The inefficient repair of DNA interstrand cross-links (ICL) is considered as the major cytogenic defect in FANCA-deficient cells [32]. The repair of ICL requires the interplay of FA, TC-NER, HR and TLS pathways [33]. Since these same pathways show differential regulation between healthy and FA-deficient $\mathrm{HIO}$, this indicates that the genetic predisposition underlying FA leads to an altered disease specific transcriptional response upon exposure to radiation. The fact that compared to healthy conditions translational synthesis (TLS) polymerases involved in tolerating replication stress-induced DNA damage [34] is downregulated in FANCA-deficient HIO further shows that the presence of FANCA is required for the regulation of REV1-, POLK- and POLI-dependent TLS pathways as previously reported [35]. In addition, several FA components and FA pathway-related genes are simultaneously downregulated in FA-deficient $\mathrm{HIO}$, indicating that that FA repair pathways fail to be activated in response to IR in FANCA defective versus healthy cells.

The importance of the observed differentially regulated pathways for the repair of ICL in FA-deficient HIO suggests that similar to the sensitivity of FA cells to mitomycin C and cisplatin, radiosensitivity in FA-deficient cells can also be defined as the failure to repair ICL. The effect of lesions related to ICL in relation to radiation-induced damage is controversial [36] at present. Our comparative analyses of FA-deficient and healthy HIO however show that genes relevant for the interplay of FA-, TC-NER- and TLS- pathways essential for the repair of ICL are actively transcribed in response to radiation in healthy versus FA-deficient $\mathrm{HIO}$, which are expected to have impaired ICL repair. This indicates that the frequency and repair of ICL may be relevant after exposure to radiation, in particular for defining the sensitivity of the GI tract for FA patients.

In this respect genes involved in the formation of TC-NER Pre-Incision Complex and coordination of dual incision in TC-NER are among the strongest factors distinguishing the transcriptional response of photon compared to proton and carbon irradiated $\mathrm{HIO} 12 \mathrm{~h}$ after radiation. This transcriptional pattern suggests that the frequency of interstrand crosslinks (ICL) which requires dual incision complexes to be repaired by TC-NER differs between photon and particle beams. On the contrary clustering analyses $48 \mathrm{~h}$ after radiation separated photon and proton from carbon gene expression patterns indicating that at later time points high LET irradiation and higher RBE induced by carbon ions leads to a unique transcriptional response including prominent DNA repair relevant factors such as SUMO3, ALC1, POLE4, PCBP4, and MUTYH. Based on the identified gene lists a core set of genes was identified which were simultaneously differentially regulated based on the FA status, radiation qualities and higher RBE. Over 50\% of these genes are involved in molecular processes regulating p53 activity and may serve as molecular surrogates resembling normal GI radio sensitivity and/or toxicity of FA patients compared to healthy patients. Further evaluation of these candidate genes is required in a larger cohort of patient-derived healthy and FA-derived HIO at different dose ranges. Based on our analyses it will be interesting to analyze DSB repair kinetics and transcriptional patterns 
in specific cell population to determine the sensitivity of different compartments and cell types in patient-derived organoids.

\section{Materials and Methods}

\subsection{Generation and Cultivation of Human Intestinal Organoids}

The HIO were derived from induced pluripotent stem cells as described previously [4]. Briefly, human induced pluripotent stem cells were grown in 6-well surface plates (Nunclon, ThermoFisher, Germany) coated with Matrigel (BD Biosciences, Germany) and maintained in mTESR1 media (Stem Cell Technologies, Germany). For induction of definitive endoderm (DE), iPS cells were passaged with Dispase (Invitrogen, Germany) and plated in a Matrigel-coated, Nunclon surface 6-well plate. $10 \mu \mathrm{M}$ Y27632 compound (Sigma Aldrich, Germany) was added to the media for the first 3 days. Cells were allowed to grow until they reached $60-70 \%$ confluence. Cells were then treated with 100 $\mathrm{ng} / \mathrm{mL}$ of Activin A for 3 days. DE was then treated with hindgut induction medium (RPMI 1640, $2 \%$ complemented FCS, $500 \mathrm{ng} / \mathrm{mL}$ rhFGF4 and $3 \mu \mathrm{M}$ Chiron99021) for 4 days to induce formation of mid-hindgut spheroids. 30-50 Spheroids were then plated in ice-cold Matrigel (BD) and maintained in intestinal growth medium (Advanced DMEM/F-12, N2, B27, 15 mM HEPES, 2 mM 1-glutamine, penicillin-streptomycin) supplemented with $50 \mathrm{ng} / \mathrm{mL}$ rhEGF (R\&D), $100 \mathrm{ng} / \mathrm{mL}$ Noggin (R\&D) and $500 \mathrm{ng} / \mathrm{mL}$ rhRSpondin1 to generate human intestinal organoids (HIOs). Media was changed at 3 days. Mature HIOs were developed approximately after 40 days. The 106 and 104 lines were cultivated in intestinal growth medium in which $100 \mathrm{ng} / \mathrm{mL}$ doxycycline was added in order to rescue the FANCA deficiency by ectopic expression of the FANCA.

\subsection{Irradiation of Human Intestinal Organoids with Photon, Proton and Carbon Irradiation}

For irradiation 2-3 HIO per well of a 96-well plate were embedded in Matrigel covered with intestinal growth medium. 96-well plates were irradiated with 4Gy photon, proton and carbon beams separately. Proton or carbon ions were administered at the Heidelberg Ion-Beam Therapy Center (HIT) as described [16,37]. For organoids which were irradiated with proton and carbon particles a $15 \mathrm{~mm}$ spread-out bragg peak (SOBP) with a middle depth of $120 \mathrm{~mm}$ was chosen. For proton beams energy ranges between 128.7-137 MeV were chosen with an average LET of $4.6 \mathrm{keV} / \mu \mathrm{m}$ ranging between $3.7-8.1 \mathrm{keV} / \mu \mathrm{m}$ in the SOBP. For carbon beams the energy range was between $2972-3152 \mathrm{MeV}$ with an average LET of $86 \mathrm{keV} / \mu \mathrm{m}$ ranging from $65-155 \mathrm{keV} / \mu \mathrm{m}$ in the SOBP.

\subsection{Immunofluorescence Staining and Microscopy of Human Intestinal Organoids}

Approximately $1 \mathrm{~h}$ after exposure to photon, proton, and carbon irradiation the HIO which were embedded in Matrigel 96-well plates were transferred in a $15 \mathrm{~mL}$ Falcon tube containing $4 \%$ paraformaldehyde while removing as much of the Matrigel as possible and gently rocked for $1 \mathrm{~h}$ at $4{ }^{\circ} \mathrm{C}$. HIO were washed three times with PBS at room temperature for $10 \mathrm{~min}$ and transferred to a $30 \%$ sucrose/PBS solution for overnight incubation at $4{ }^{\circ} \mathrm{C}$. The HIO were then transferred to the center of an embedding mold and covered with Cryo-OCT and incubated at room temperature for 30 min. A dry ice/ $70 \%$ ethanol mixture was prepared, and embedded molds were placed in this mixture until the OCT turned completely white. For long-term storage the embedded molds were placed at $-80{ }^{\circ} \mathrm{C}$ before cryosection was performed. For cryosection the OCT blocks were warmed up for approximately $15 \mathrm{~min}$ to reach a temperature of $-20^{\circ} \mathrm{C}$. Sections were cut and placed on microscopy slides for subsequent immune staining of radiation-induced DSB as described (PMID: 26254681, PMID: 27494855). Briefly, Irradiated HIO, slides were washed twice with PBS and blocked 30 min with $3 \%$ BSA in PBS+0.1\% Triton. The p53-binding protein 1 (53BP1) polyclonal antibody (Cell Signaling; 4937) was prepared at a dilution of 1:200 and incubated over night at $4{ }^{\circ} \mathrm{C}$ in a humid incubation chamber. Slides were washed three times in PBS and incubating with $5 \mu \mathrm{g} / \mathrm{mL}$ goat anti-rabbit Alexa-555 for $1 \mathrm{~h}$ at room temperature in the dark. Slides were washed again for 3 times with PBS and 1 time in ddH2O 
and mounted with DAPI mounting reagent (life technologies; P36931). Microscopy and imaging were done with Olympus R cell microscopy at the DKFZ core facility. For the quantification of 53BP1-foci numbers and area the $40 \times$ magnifications were used and the foci numbers and are counted with the ImageJ software. In parallel the are values of DAPI were quantified to determine the cell number in each image. Values for foci numbers were normalized to the DAPI area of each image to determine foci numbers and area per cell. These values were then normalized to the values obtained for 0Gy to calculate the fold change of 53BP1 numbers and size in $\mathrm{HIO}$ after exposure to photon, proton and carbon irradiation.

\subsection{Isolation of RNA from Human Intestinal Organoids}

$\mathrm{HIO}$ were frozen 12 and $48 \mathrm{~h}$ after irradiation in RLT buffer (Quiagen 79216) and stored at $-80{ }^{\circ} \mathrm{C}$ before RNA was isolated. For RNA isolation, the HIO were homogenized and total RNA was isolated using the RNeasy Mini Kit (Qiagen, Germany) according to the manufactures protocol followed by quality control performed by Bioanalyzer and quantification by nanodrop. For genome-wide expression analyses Illumina HumanHT-12 v4 Expression BeadChip Kit was utilized and the microarray hybridization and scanning were performed at $\mathrm{dkfz}$ core facility.

\subsection{Analysis of Gene Expression Values}

Gene-expression analysis was performed as described [15,38] Gene transcripts with 30\% or more non-assessable reads on the microarray were excluded from the analysis. Total norm intensity was performed on all samples. To further correct for background-noise from the microarray readout, only genes with a mean intensity-level of $\geq 100$ were included in the analysis. The gene expression data of irradiated $\mathrm{HIO}$ were normalized to the arithmetic mean values of the respective control-groups. To identify differentially regulated genes ANOVA, two-class unpaired $t$-test, and multiple testing correction (Bonferroni) was applied using the in-house developed Software (write out first time) SUMO, version 1.61j [38]. Hierarchical clustering using Manhatton distance (average sistance) was performed with SUMO. Microarray data is available online at ArrayExpress [39].

\section{Conclusions}

This study shows that gene expression analyses of pluripotent derived human intestinal organoids proves feasible for distinguishing transcriptional programs corresponding to normal radiosensitvity modeled by $\mathrm{HIO}$ of healthy patients compared to highly radiation sensitive tissues modeled by $\mathrm{HIO}$ of FA patients. Interestingly unique gene expression signatures after exposure of HIO to photon, proton and carbon irradiation were identified which indicate a possible difference in the frequency of interstrand crosslinks induced by photon and particle beams. Combined with the identified differential gene expression signatures of three different radiation qualities, and genes correlating to increased RBE induced by high LET carbon irradiation, candidate molecular surrogates of normal tissue radiotoxicity in FA patients compared to healthy conditions have been identified. These may prove clinically relevant for determining FA patient radio- sensitivity and/or toxicity doses for photon, proton and carbon ions.

Supplementary Materials: The following are available online at http://www.mdpi.com/2072-6694/12/2/469/s1. Figure S1: A local effect model (LEM) predicting RBE doses of proton and carbon compared to photon; Figure S2: Gene expression differences between 104-HIO and 106-HIO FANCA rescued and OFF. Figure S3: Gene expression differences of FANCA proficient and deficient HIO in comparison to wtHIO; Figure S4: STRING (Search Tool for the Retrieval of Interacting Genes/Proteins) analyses of Protein-Protein interaction networks of genes specifically deregulated in FANCA deficient HIO; Figure S5: Gene expression regulation patterns after 12 and $48 \mathrm{~h}$ post IR; Figure S6: Genes sequentially regulated after 12 and $48 \mathrm{~h}$ in response to photon, proton and carbon irradiation; Figure S7: One-way-ANOVA analyses of gene expression values of photon, proton and carbon beams; Figure S8: Two-way-ANOVA analyses to assess the interaction of the FA and radiation quality effect on irradiated HIO with no functionally active FA pathway; Table S1: One-way-ANOVA between wtHIO, 106-HIO FANCA rescued and 106-HIO FANCA deficient; Table S2: Genes differentially expressed between 106-HIO FANCA deficient compared to H1-HIO (T-test); Table S3: DNA damage response genes differentially expressed between 106-HIO FANCA rescued and 106-HIO FANCA deficient ( $t$-test); Table S4: Reactome analyses of DDR Genes differentially expressed 
in 106-HIO FANCA deficient compared to 106-HIO FANCA rescued; Table S5: One-way-ANOVA for identifying genes differentially expressed after Photon, Proton and Carbon irradiation in all HIO. Table S6: Genes who's expression correlate or anti-correlate with the higher RBE of Carbon ions; Table S7: genes differentially regulated in response to the different radiation qualities and the FANCA expression status (two-way-ANOVA); Table S8: Common genes differentially regulated $12 \mathrm{~h}$ post exposure to photon, proton and carbon irradiation ( $T$-test) and Reactome analyses of genes differentially regulated in HIO irradiated with photon, proton and carbon; TableS9: Reactome analyses of genes upregulated in HIO irradiated with photon, proton and carbon after $12 \mathrm{~h}$; Table S10: Reactome analyses of genes downregulated in HIO irradiated with photon, proton and carbon after $12 \mathrm{~h}$; Table S11: Common genes differentially regulated $48 \mathrm{~h}$ post exposure to photon, proton and carbon irradiation (T-test) and Reactome analyses of genes differentially regulated in HIO irradiated with photon, proton and carbon. Table S12: Reactome analyses of genes upregulated in HIO irradiated with photon, proton and carbon after $48 \mathrm{~h}$. Table S13: Reactome analyses of genes downregulated in HIO irradiated with photon, proton and carbon after $48 \mathrm{~h}$; Table S14: Reactome analyses and Genes differentially regulated between 12 and $48 \mathrm{~h}$ post exposure to photon, proton and carbon irradiation in all HIO (T-test); Table S15: Overview of irradiated pluripotent derived human intestinal organoids.

Author Contributions: A.N., M.G.S., S.I.W., J.M.W. and A.A. conceived the project. M.K. (Matthew Kuhar) generated the human intestinal organoids. A.N. irradiated the HIO and prepared the samples for IF staining and microarray analyses. M.A. stained the HIO and performed analyses of the radiation-induced foci. M.K. (Maximillian Knoll) assisted on quantifying the radiation-induced foci. D.K. wrote the ImageJ macros and provided valuable advise on analyzing the radiation-induced foci data. A.N. analyzed the gene expression data. C.S. provided valuable advice for the gene expression analyses. S.B. made the particle therapy radiation plans. A.N. prepared and wrote the manuscript. A.A., S.I.W., J.M.W. and J.D. provided valuable discussion and conceptual advice and edited the manuscript. All authors have read and agreed to the published version of the manuscript.

Funding: This work was supported by the German Research Foundation (DFG, KFO-214), German Krebshilfe (Deutsche Krebshilfe, Max-Eder 108876) and intramural funds of the National Center for Tumor Diseases (NCT3.0_2015.21/22 NCT-PRO and Biodose programs).

Acknowledgments: This work was conducted in frame of German Research Foundation (DFG) "clinical research group heavy ion therapy (KFO-214)" within the central platform (ZP1). For technical assistance, we are grateful to Claudia Rittmüller and Barbara Schwager. We thank Peter Haering and Mona Splinter for preparing the radiation plans for Photon irradiation. We are grateful to the Heidelberg Ion-Beam Therapy Center for providing beam time to irradiated the HIO. We thank the microarray unit of the DKFZ Genomics and Proteomics Core Facility for providing the Illumina Whole-Genome Expression Beadchips and related services. We thank the DKFZ Light Microscopy Facility for support.

Conflicts of Interest: The authors declare no conflict of interest.

\section{Appendix A}

Common transcriptional responses after 12 and $48 \mathrm{~h}$ in $\mathrm{HIO}$ which where irradiated by photon, proton and carbon radiation. $12 \mathrm{~h}$ post IR a total of 781 genes (Supplementary Table S8) were identified which were significantly $(p<0.01)$ and commonly de-regulated in all three radiation qualities. Reactome pathway analyses revealed that $12 \mathrm{~h}$ after irradiation mainly genes and pathways (Supplementary Table S9) involved in the transcriptional regulation by TP53 were upregulated. Genes related to ATR-mediated stress response and DNA replication such as the origin recognition complex genes (ORC3L, ORC4L, ORC6L) and mini-chromosome maintenance (MCM6 and MCM8) as well as other genes which regulate DNA replication (RPA3) and the ATR stress response were downregulated at the $12 \mathrm{~h}$ time point (Supplementary Table S10). Gene expression analyses after $48 \mathrm{~h}$ resulted in 632 significantly $(p<0.01)$ de-regulated genes (Supplementary Table S11). In comparison to gene expression changes $12 \mathrm{~h}$ post IR, the upregulation of genes involved in the regulation by TP53 is still maintained (Supplementary Table S12) after $48 \mathrm{~h}$. The genes which were downregulated $48 \mathrm{~h}$ post irradiation (Supplementary Table S13) were predominantly involved in the regulation of the cell cycle, however compared to $12 \mathrm{~h}$ the intensity and significance of downregulated genes regulating cell cycle checkpoints was more dominant (Supplementary Figure S5). Especially mitotic spindle checkpoint and separation of duplicated chromosomes defined by the downregulation of Mitotic Checkpoint Serine/Threonine Kinases and proteins (BUB1, BUB1B, BUB3), centromere proteins (CENPA, CENPE, CENPK, CENPL, CENPM) and components of the anaphase promoting complex/cyclosome (APC/C) (CDCA4, CDCA5, CDCA8, CDC16, CDC20, CDC25C, CDC45L) were downregulated specifically after $48 \mathrm{~h}$. Also genes involved in the regulation of DNA replication defined by the highly conserved mini-chromosome maintenance proteins (MCM) (MCM2, MCM3, MCM4, MCM5, MCM6, MCM8) 
were downregulated $48 \mathrm{~h}$ post radiation. The majority of the up or downregulated genes were time point specific with minimal overlap between both time points (Supplementary Figure S5). Gene expression alterations are more significant $48 \mathrm{~h}$ post IR since the number of functional categories and pathways affected by the gene expression changes were higher compared to the $12 \mathrm{~h}$ time point (Supplementary Figure S5). 15 of a total of 110 (12 h 25, 48 h 86) functional reactome categories affected by the IR-induced gene expression alterations overlapped between the 12 and $48 \mathrm{~h}$ timepoints (Supplementary Figure S5).

\section{Appendix B}

Sequential gene expression changes of irradiated HIO reveal functional alterations in response to photon, proton and carbon radiation. To exclusively analyze the differences between both time points independent of the common transcriptional response to IR described above we compared gene expression signatures of 12 and $48 \mathrm{~h}$ post IR after virtual pool normalization instead of normalizations to non-irradiated controls. A total of 1190 genes (Supplementary Table S14) were identified to be significantly $(p<0.01, T$-test Bonferroni corrected) differentially regulated between 12 and $48 \mathrm{~h}$ in all of the irradiated HIO. A sequential gene expression signature was detected with genes regulation eukaryotic translation. The L-ribosomal assembly proteins (RPL6, RPL7A, RPL8, RPL10A, RPL15, RPL19, RPL22, RPL26L1, RPL29, RPL36A, RPL39L, RPLP0), the S-ribosomal assembly proteins (RPS17, RPS4X, RPS5) and the translation initiation factors EEF1B2 and EIF2B2 (Supplementary Figure S6) had a significantly higher expression value $12 \mathrm{~h}$ compared to $48 \mathrm{~h}$ after exposure to IR. Also the mitochondrial L and S ribosomal genes (MRPL3, MRPL9, MRPL18, MRPL30, MRPL36, MRPL39, MRPL40, MRPL45, MRPL49, MRPL51, MRPS15, MRPS21, MRPS22, MRPS23, MRPS28, MRPS30, MRPS31, MRPS33) were sequentially regulated in the same direction (Supplementary Figure S6) indicating a sequential downregulation of mitochondrial translation in response to IR. This was accompanied by a third sequential regulation pattern which involves the ubiquitin-mediated proteasome degradation pathway (Supplementary Figure S5) which is regulated in the anaphase-promoting complex/cyclosome (APC/C) by the proteinase complex subunits (PSMA1, PSMA3, PSMA5, PSMA6, PSMB7, PSMB8, PSMC2, PSMC4, PSMC6, PSMD6, PSMD10, PSMD12, PSMD14, PSMG2) which were all higher expressed at 12 $\mathrm{h}$ compared to $48 \mathrm{~h}$. The only functional category which was specifically upregulated $48 \mathrm{~h}$ post IR were defined by genes involved in regulating PPARalpha-mediated lipid metabolism.

\section{References}

1. Bentzen, S.M.; Constine, L.S.; Deasy, J.O.; Eisbruch, A.; Jackson, A.; Marks, L.B.; Ten Haken, R.K.; Yorke, E.D. Quantitative Analyses of Normal Tissue Effects in the Clinic (QUANTEC): an introduction to the scientific issues. Int. J. Radiat. Oncol. Biol. Phys. 2010, 76 (Suppl. 3), S3-S9. [CrossRef] [PubMed]

2. Bentzen, S.M. Preventing or reducing late side effects of radiation therapy: radiobiology meets molecular pathology. Nat. Rev. Cancer 2006, 6, 702-713. [CrossRef] [PubMed]

3. Francois, A.; Milliat, F.; Guipaud, O.; Benderitter, M. Inflammation and immunity in radiation damage to the gut mucosa. Biomed. Res. Int. 2013, 2013, 123241. [CrossRef] [PubMed]

4. Shadad, A.K.; Sullivan, F.J.; Martin, J.D.; Egan, L.J. Gastrointestinal radiation injury: symptoms, risk factors and mechanisms. World J. Gastroenterol. 2013, 19, 185-198. [CrossRef] [PubMed]

5. Olcina, M.M.; Giaccia, A.J. Reducing radiation-induced gastrointestinal toxicity-The role of the PHD/HIF axis. J. Clin. Investig. 2016, 126, 3708-3715. [CrossRef] [PubMed]

6. McCracken, K.W.; Howell, J.C.; Wells, J.M.; Spence, J.R. Generating human intestinal tissue from pluripotent stem cells in vitro. Nat. Protoc. 2011, 6, 1920-1928. [CrossRef]

7. Spence, J.R.; Mayhew, C.N.; Rankin, S.A.; Kuhar, M.F.; Vallance, J.E.; Tolle, K.; Hoskins, E.E.; Kalinichenko, V.V.; Wells, S.I.; Zorn, A.M.; et al. Directed differentiation of human pluripotent stem cells into intestinal tissue in vitro. Nature 2011, 470, 105-109. [CrossRef]

8. Wells, J.M.; Spence, J.R. How to make an intestine. Development 2014, 141, 752-760. [CrossRef]

9. Clevers, H. Modeling Development and Disease with Organoids. Cell 2016, 165, 1586-1597. [CrossRef] 
10. Takebe, T.; Wells, J.M.; Helmrath, M.A.; Zorn, A.M. Organoid Center Strategies for Accelerating Clinical Translation. Cell Stem Cell 2018, 22, 806-809. [CrossRef]

11. Dutta, D.; Heo, I.; Clevers, H. Disease Modeling in Stem Cell-Derived 3D Organoid Systems. Trends. Mol. Med. 2017, 23, 393-410. [CrossRef]

12. Sachs, N.; de Ligt, J.; Kopper, O.; Gogola, E.; Bounova, G.; Weeber, F.; Balgobind, A.V.; Wind, K.; Gracanin, A.; Begthel, H.; et al. A Living Biobank of Breast Cancer Organoids Captures Disease Heterogeneity. Cell 2018, 172, 373-386. [CrossRef] [PubMed]

13. Weeber, F.; Ooft, S.N.; Dijkstra, K.K.; Voest, E.E. Tumor Organoids as a Pre-clinical Cancer Model for Drug Discovery. Cell Chem. Biol. 2017, 24, 1092-1100. [CrossRef] [PubMed]

14. Zhou, C.; Jones, B.; Moustafa, M.; Schwager, C.; Bauer, J.; Yang, B.; Cao, L.; Jia, M.; Mairani, A.; Chen, M.; et al. Quantitative assessment of radiation dose and fractionation effects on normal tissue by utilizing a novel lung fibrosis index model. Radiat. Oncol. 2017, 12, 172. [CrossRef] [PubMed]

15. Zhou, C.; Moustafa, M.R.; Cao, L.; Kriegsmann, M.; Winter, M.; Schwager, C.; Jones, B.; Wang, S.; Bauerle, T.; Zhou, P.K.; et al. Modeling and multiscale characterization of the quantitative imaging based fibrosis index reveals pathophysiological, transcriptome and proteomic correlates of lung fibrosis induced by fractionated irradiation. Int. J. Cancer 2019, 144, 3160-3173. [CrossRef]

16. Klein, C.; Dokic, I.; Mairani, A.; Mein, S.; Brons, S.; Haring, P.; Haberer, T.; Jakel, O.; Zimmermann, A.; Zenke, F; et al. Overcoming hypoxia-induced tumor radioresistance in non-small cell lung cancer by targeting DNA-dependent protein kinase in combination with carbon ion irradiation. Radiat. Oncol. 2017, 12, 208. [CrossRef]

17. Chlon, T.M.; Ruiz-Torres, S.; Maag, L.; Mayhew, C.N.; Wikenheiser-Brokamp, K.A.; Davies, S.M.; Mehta, P.; Myers, K.C.; Wells, J.M.; Wells, S.I. Overcoming Pluripotent Stem Cell Dependence on the Repair of Endogenous DNA Damage. Stem Cell Rep. 2016, 6, 44-54. [CrossRef]

18. Kutler, D.I.; Patel, K.R.; Auerbach, A.D.; Kennedy, J.; Lach, F.P.; Sanborn, E.; Cohen, M.A.; Kuhel, W.I.; Smogorzewska, A. Natural history and management of Fanconi anemia patients with head and neck cancer: A 10-year follow-up. Laryngoscope 2016, 126, 870-879. [CrossRef]

19. Raya, A.; Rodriguez-Piza, I.; Guenechea, G.; Vassena, R.; Navarro, S.; Barrero, M.J.; Consiglio, A.; Castella, M.; Rio, P.; Sleep, E.; et al. Disease-corrected haematopoietic progenitors from Fanconi anaemia induced pluripotent stem cells. Nature 2009, 460, 53-59. [CrossRef]

20. Muller, L.U.; Milsom, M.D.; Harris, C.E.; Vyas, R.; Brumme, K.M.; Parmar, K.; Moreau, L.A.; Schambach, A.; Park, I.H.; London, W.B.; et al. Overcoming reprogramming resistance of Fanconi anemia cells. Blood 2012, 119, 5449-5457. [CrossRef]

21. Galanty, Y.; Belotserkovskaya, R.; Coates, J.; Polo, S.; Miller, K.M.; Jackson, S.P. Mammalian SUMO E3-ligases PIAS1 and PIAS4 promote responses to DNA double-strand breaks. Nature 2009, 462, 935-939. [CrossRef] [PubMed]

22. Ahel, D.; Horejsi, Z.; Wiechens, N.; Polo, S.E.; Garcia-Wilson, E.; Ahel, I.; Flynn, H.; Skehel, M.; West, S.C.; Jackson, S.P.; et al. Poly(ADP-ribose)-dependent regulation of DNA repair by the chromatin remodeling enzyme ALC1. Science 2009, 325, 1240-1243. [CrossRef] [PubMed]

23. Bellelli, R.; Belan, O.; Pye, V.E.; Clement, C.; Maslen, S.L.; Skehel, J.M.; Cherepanov, P.; Almouzni, G.; Boulton, S.J. POLE3-POLE4 Is a Histone H3-H4 Chaperone that Maintains Chromatin Integrity during DNA Replication. Mol. Cell 2018, 72, 112-126 e5. [CrossRef] [PubMed]

24. Zhu, J.; Chen, X. MCG10, a novel p53 target gene that encodes a KH domain RNA-binding protein, is capable of inducing apoptosis and cell cycle arrest in G(2)-M. Mol. Cell Biol. 2000, 20, 5602-5618. [CrossRef] [PubMed]

25. Scoumanne, A.; Cho, S.J.; Zhang, J.; Chen, X. The cyclin-dependent kinase inhibitor p21 is regulated by RNA-binding protein PCBP4 via mRNA stability. Nucleic Acids Res. 2011, 39, 213-224. [CrossRef]

26. Oka, S.; Nakabeppu, Y. DNA glycosylase encoded by MUTYH functions as a molecular switch for programmed cell death under oxidative stress to suppress tumorigenesis. Cancer Sci. 2011, 102, 677-682. [CrossRef]

27. Mikhailov, A.; Cole, R.W.; Rieder, C.L. DNA damage during mitosis in human cells delays the metaphase/anaphase transition via the spindle-assembly checkpoint. Curr. Biol. 2002, 12, 1797-1806. [CrossRef]

28. Peters, J.M. The anaphase-promoting complex: proteolysis in mitosis and beyond. Mol. Cell 2002, 9, 931-943. [CrossRef] 
29. Peters, J.M. The anaphase promoting complex/cyclosome: a machine designed to destroy. Nat. Rev. Mol. Cell Biol. 2006, 7, 644-656. [CrossRef]

30. Molina-Estevez, F.J.; Nowrouzi, A.; Lozano, M.L.; Galy, A.; Charrier, S.; von Kalle, C.; Guenechea, G.; Bueren, J.A.; Schmidt, M. Lentiviral-Mediated Gene Therapy in Fanconi Anemia-A Mice Reveals Long-Term Engraftment and Continuous Turnover of Corrected HSCs. Curr. Gene Ther. 2015, 15, 550-562. [CrossRef]

31. Sondalle, S.B.; Longerich, S.; Ogawa, L.M.; Sung, P.; Baserga, S.J. Fanconi anemia protein FANCI functions in ribosome biogenesis. Proc. Natl. Acad. Sci. USA 2019, 116, 2561-2570. [CrossRef] [PubMed]

32. Duxin, J.P.; Walter, J.C. What is the DNA repair defect underlying Fanconi anemia? Curr. Opin. Cell Biol. 2015, 37, 49-60. [CrossRef]

33. Deans, A.J.; West, S.C. DNA interstrand crosslink repair and cancer. Nat. Rev. Cancer 2011, 11, 467-480. [CrossRef]

34. Waters, L.S.; Minesinger, B.K.; Wiltrout, M.E.; D'Souza, S.; Woodruff, R.V.; Walker, G.C. Eukaryotic translesion polymerases and their roles and regulation in DNA damage tolerance. Microbiol. Mol. Biol. Rev. 2009, 73, 134-154. [CrossRef] [PubMed]

35. Kim, H.; Yang, K.; Dejsuphong, D.D.; Andrea, A.D. Regulation of Rev1 by the Fanconi anemia core complex. Nat. Struct. Mol. Biol. 2012, 19, 164-170. [CrossRef] [PubMed]

36. Dextraze, M.E.; Gantchev, T.; Girouard, S.; Hunting, D. DNA interstrand cross-links induced by ionizing radiation: an unsung lesion. Mutat. Res. 2010, 704, 101-107. [CrossRef] [PubMed]

37. Chiblak, S.; Tang, Z.; Lemke, D.; Knoll, M.; Dokic, I.; Warta, R.; Moustafa, M.; Mier, W.; Brons, S.; Rapp, C.; et al. Carbon irradiation overcomes glioma radioresistance by eradicating stem cells and forming an antiangiogenic and immunopermissive niche. JCI Insight 2019, 4, e123837. [CrossRef]

38. Melzig, C.; Golestaneh, A.F.; Mier, W.; Schwager, C.; Das, S.; Schlegel, J.; Lasitschka, F.; Kauczor, H.U.; Debus, J.; Haberkorn, U.; et al. Combined external beam radiotherapy with carbon ions and tumor targeting endoradiotherapy. Oncotarget 2018, 9, 29985-30004. [CrossRef]

39. Athar, A.; Fullgrabe, A.; George, N.; Iqbal, H.; Huerta, L.; Ali, A.; Snow, C.; Fonseca, N.A.; Petryszak, R.; Papatheodorou, I.; et al. ArrayExpress update-From bulk to single-cell expression data. Nucleic Acids Res. 2019, 47, D711-D715. [CrossRef]

(C) 2020 by the authors. Licensee MDPI, Basel, Switzerland. This article is an open access article distributed under the terms and conditions of the Creative Commons Attribution (CC BY) license (http://creativecommons.org/licenses/by/4.0/). 